Reprinted from:

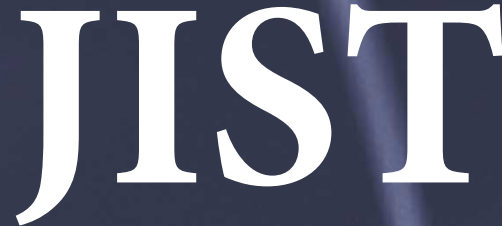

Vol. 53, No. 5 September/October 2009
Journal of

Imaging Science

\section{and Technology}

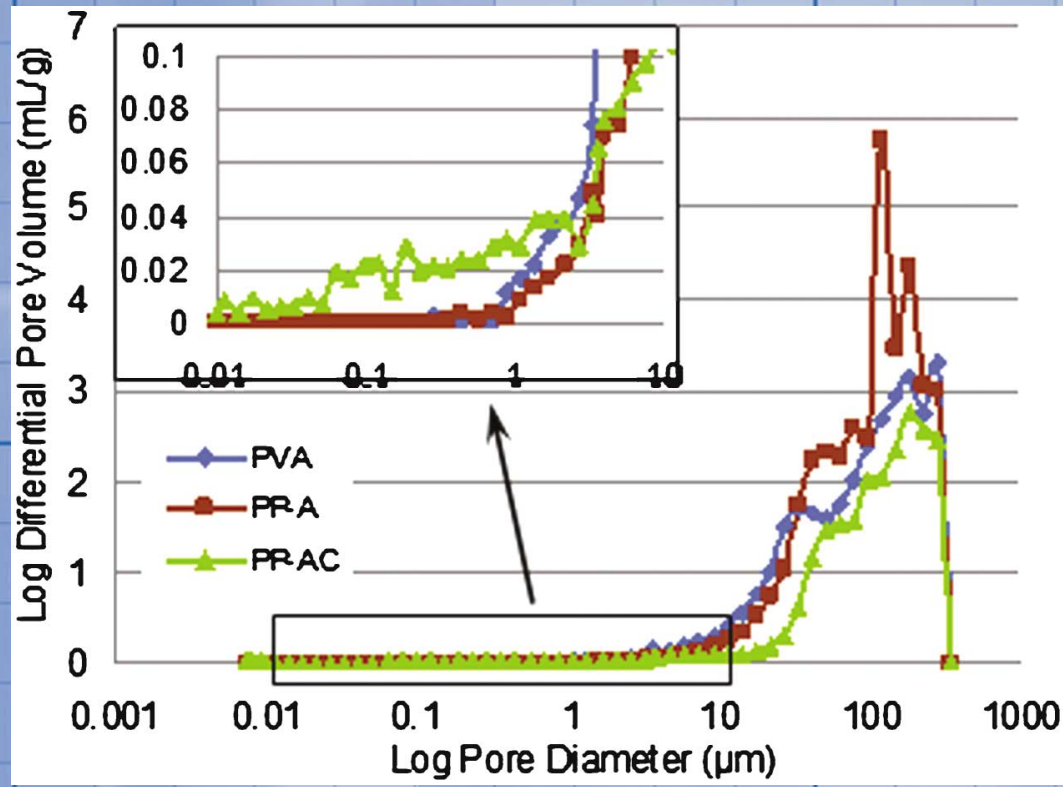

Society for Imaging Science and Technology 



\title{
Crystal Structure of Methyl Orange Derivatives and Their Electronic Spectra
}

\author{
H. Shibata, K. Sato and J. Mizuguchi ${ }^{\wedge}$ \\ Graduate School of Engineering, Yokohama National University, 240-8501 Yokohama, Japan \\ E-mail: mizu-j@ynu.ac.jp
}

\begin{abstract}
Azo dyes and pigments are widely used in the imaging industry because of their versatile colors as well as their low price. However, it is often pointed out that the color in the solid state is sometimes drastically different from that in solution. An attempt has been therefore made in the present investigation to elucidate the electronic structure of two classical simple azo dyes on the basis of the crystal structure: methyl orange [Na(I)-4' (dimethylamino)azobenzene-4-sulfonate (MO)] and its derivative [4-(dimethylamino)azobenzene-4' -sulfonic acid (MOH)]. Accordingly the authors found a new trinuclear Na complex (pale red) of $\mathrm{MO}$, as well as a new polymorph (purple) of $\mathrm{MOH}$ in addition to the previously reported phase (vivid red). In MO and also in the purple phase of $\mathrm{MOH}$, excitonic interactions are basically responsible for the color change on going from solution to the solid state. On the other hand, the authors found a zwitterionic structure in the vivid-red phase of $\mathrm{MOH}$ as formed by $\mathrm{NH} \cdots \mathrm{O}$ intermolecular hydrogen bonds. The vivid-red color can be interpreted as arising mainly from the zwitterionic structure. () 2009 Society for Imaging Science and Technology.

[DOI: 10.2352/J.ImagingSci.Technol.2009.53.5.050302]
\end{abstract}

\section{INTRODUCTION}

Azo dyes and pigments are widely used in the imaging industry because of their versatile colors as well as their low price. The azo color is considered to arise from the azo group $(-\mathrm{N}=\mathrm{N}-)$, as exemplified by methyl orange and its derivative (i.e., the title compounds): $\mathrm{Na}(\mathrm{I})-4^{\prime}$ (dimethylamino)azobenzene-4-sulfonate [abbreviated as MO: Figure 1(a)] and 4-(dimethylamino)azobenzene$4^{\prime}$-sulfonic acid [MOH: Fig. 1(b)]. As shown in Fig. 1(a), the chromogen is composed of the azo chromophore (i.e., phenyl/azo/phenyl moiety) and the auxochromes of $-\mathrm{N}\left(\mathrm{CH}_{3}\right)_{2}$ (donor: push) and of $-\mathrm{SO}_{3} \mathrm{Na}$ or $-\mathrm{SO}_{3} \mathrm{H}$ (acceptor: pull). The present system is typically called a "pushpull" system. As for the azo dye color in the solid state, it is often pointed out that the color in the solid state is sometimes drastically different from that in solution or the solidstate color depends greatly on the crystalline or amorphous state. There are also controversial reports on the azo structure suggesting that some types of azo pigments possess the hydrazone or hydrazonelike structure $(=\mathrm{N}-\mathrm{NH}-)$ rather

\section{IS\&T Member.}

Received Dec. 16, 2008; accepted for publication Jun. 17, 2009; published online Aug. 27, 2009.

$1062-3701 / 2009 / 53(5) / 050302 / 7 / \$ 20.00$.

than the azo form, ${ }^{1}$ e.g., azo pigments derived from $\beta$-naphthol, ${ }^{2,3}$ acetoacetanilide, ${ }^{4}$ and pyrazolone. ${ }^{5}$

In view of the present situation, we focused our attention on $\mathrm{MO}$ and $\mathrm{MOH}$ in order to study the correlation between the crystal structure and the color in the solid state. The present research provides valuable information on how the intermolecular interaction affects the color in the solid state and helps us to design an appropriate azo dyestuff for specific applications.

In the course of our investigation on the crystal structure of $\mathrm{MO}$ and $\mathrm{MOH}$, we found a new trinuclear azo-Na complex (pale red) of $\mathrm{MO}^{6}{ }^{6}$ as well as a new polymorph (purple) of MOH. The structures of solvated MO have previously been reported by Hanson ${ }^{7}$ and Kennedy et al. ${ }^{8}$ In these complexes, the solvent molecules served as ligands to the central $\mathrm{Na}$ atom. On the other hand, the vivid-red phase of $\mathrm{MOH}$ (vivid red) has previously been reported by Burke et al. ${ }^{9}$ The authors were, however, uniquely interested in the formation of intermolecular hydrogen bonds in $\mathrm{MOH}$, not in the color generation mechanism. In other words, we noticed that this phase possesses a zwitterionic structure $\left({ }^{-} \mathrm{O}_{3} \mathrm{SC}_{6} \mathrm{H}_{4} \mathrm{~N}^{+} \mathrm{H}=\mathrm{NC}_{6} \mathrm{H}_{4} \mathrm{NMe}_{2}\right)$ as shown in Figure 2 [see also Figure 3(d)], where the $\mathrm{H}$ atom of the sulfonic group $(\mathrm{H} 1 \mathrm{~N})$ is transferred to the $\mathrm{N} 1$ atom to form the $\mathrm{NH} \cdots \mathrm{O}$ intermolecular hydrogen bond. That is, the molecular structure in the solid state is obviously different from the structure as drawn in Fig. 1(b).

The purpose of the present investigation is to characterize the electronic structure of the tri-nuclear azo-Na complex of MO as well as of the two phases of $\mathrm{MOH}$, with special attention to the zwitterionic structure of $\mathrm{MOH}$.

\section{EXPERIMENTAL}

Crystal Growth of MO and $\mathrm{MOH}$ and Their Structure Analysis

MO was purchased from Junsei Chemical Co., Ltd., Japan. Single crystals were grown by recrystallization from a

(a)

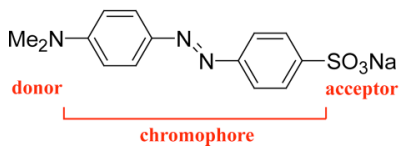

Figure 1. Molecular structure: (a) $\mathrm{MO}$ and (b) $\mathrm{MOH}$. The direction of the transition dipole $(\mu)$ as deduced from molecular orbital calculations points along the long-molecular axis in both $\mathrm{MO}$ and $\mathrm{MOH}$. 


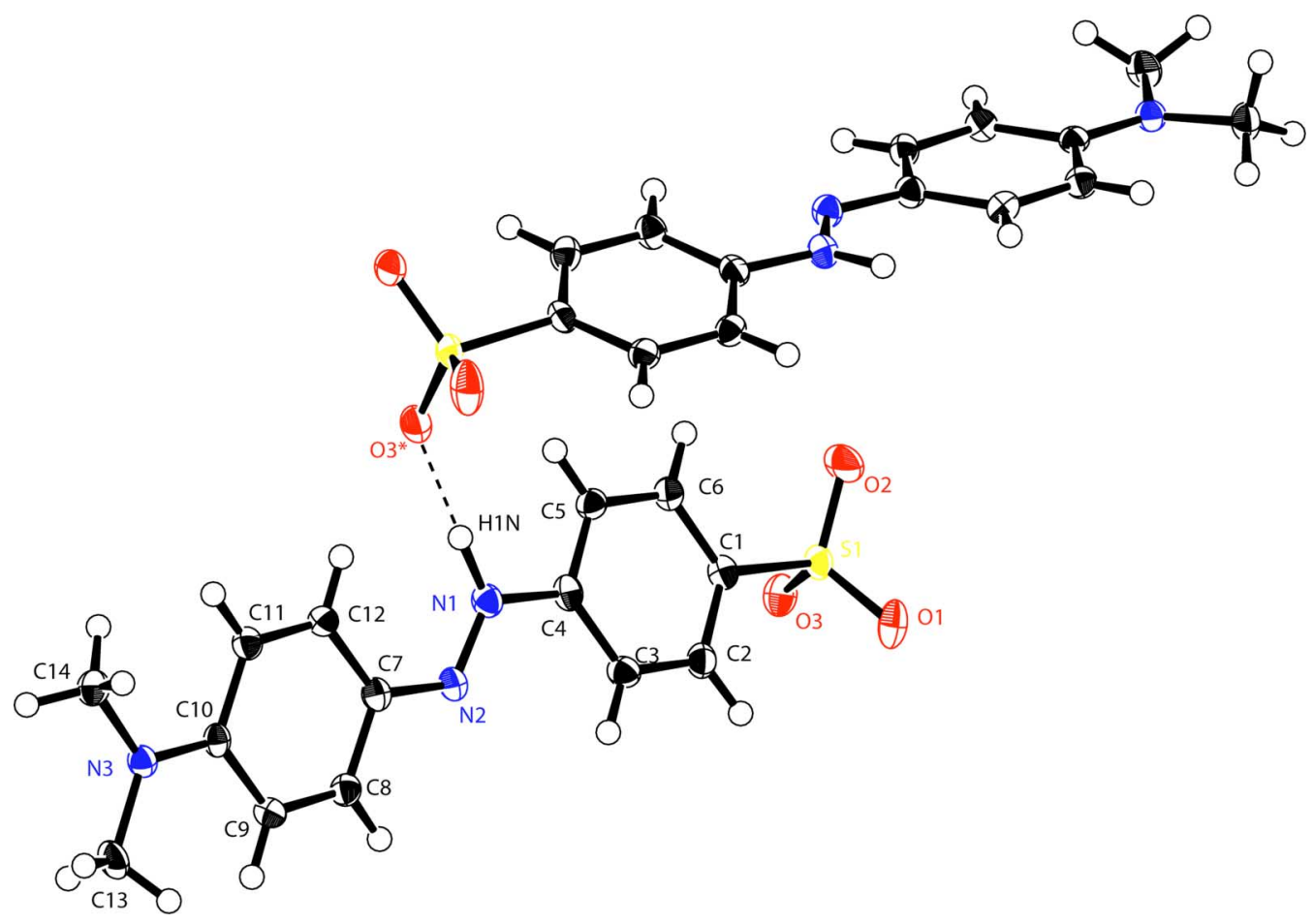

Figure 2. ORTEP plot of $\mathrm{MOH}$ reported by Burke et al. (Ref. 9) showing a zwitterionic structure: $\mathrm{NI}^{+}$and $\mathrm{SO}_{3}^{-}$.

dimethylacetamide solution by slow diffusion of 1,4-dioxane. After a week, a number of orange needlelike single crystals were obtained. Since the crystal was found to include solvent molecules, the collection of reflection data was carried out at $93 \mathrm{~K}$.

Single crystals of $\mathrm{MOH}$ were grown from an aqueous solution. After $72 \mathrm{~h}$, a number of red platelet single crystals as well as purple needlelike single crystals were obtained in the same pot at the same time. The red crystal is found to correspond to the structure reported by Burke et al. ${ }^{9}$ whereas the purple one is a new polymorph. Reflection data were collected at $93 \mathrm{~K}$ since the purple crystal was found to contain water molecules.

Table I. Crystallographic parameters for dioxane-coordinated MO.

\begin{tabular}{lc}
\hline & Methyl orange $^{\mathrm{a}}$ \\
\hline Molecular formula & $\mathrm{C}_{42} \mathrm{H}_{42} \mathrm{~N}_{9} \mathrm{O}_{9} \mathrm{~S}_{3} \mathrm{Na}_{3} \cdot \mathrm{C}_{12} \mathrm{H}_{24} \mathrm{O}_{6}$ \\
Molecular weight & 1246.34 \\
Crystal system & 0 rthorhombic \\
Space group & $P 2_{1} 2_{1} 2_{1}$ \\
$Z$ & 4 \\
$a(\AA)$ & $8.4471(6)$ \\
$b(\AA)$ & $15.5153(10)$ \\
$c(\AA)$ & $44.488(3)$ \\
$V\left(\AA^{3}\right)$ & $5830.6(7)$ \\
$D_{x}\left(\mathrm{~g} / \mathrm{cm}^{3}\right)$ & 1.491 \\
$R_{1}$ & 0.0448 \\
\hline$\left.{ }_{\mathrm{O}}{ }_{\mathrm{Re}}\right)$ &
\end{tabular}




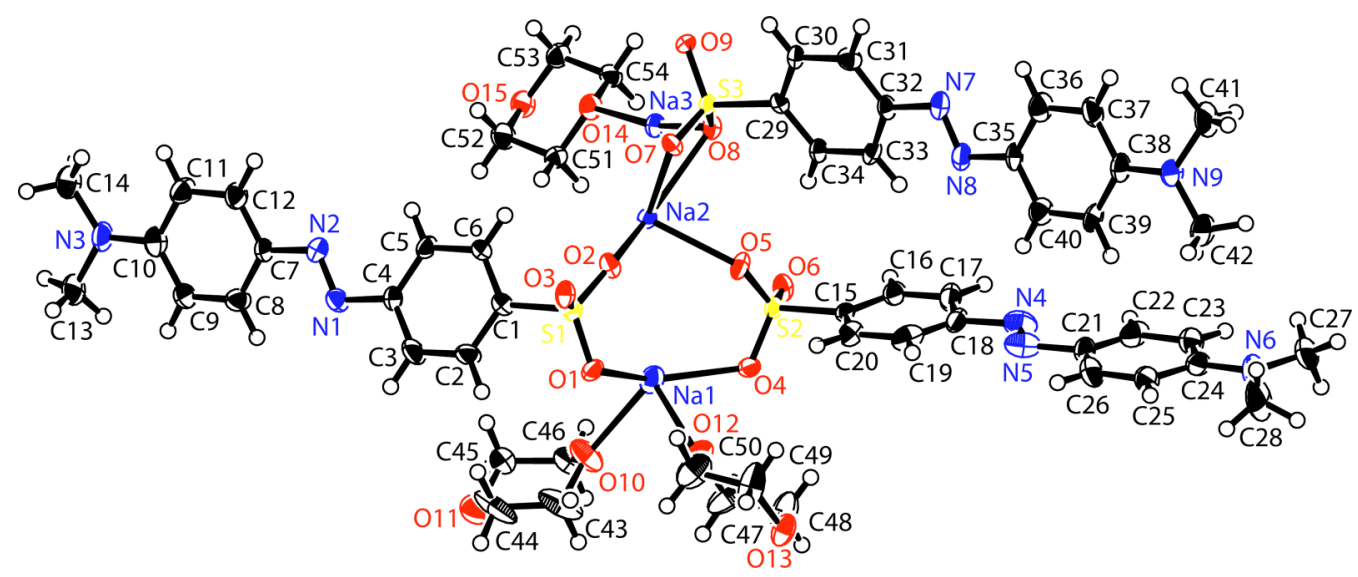

Figure 4. A view of the monomeric structure of dioxane-coordinated $M O$, showing $50 \%$ displacement ellipsoids.

under $\mathrm{N}_{2}$ (degassed). Both deprotonation and protonation processes were monitored by changes in both electrical conductivity of the solution ("conductometric titration") and absorption spectra.

\section{Density-functional Theory Calculations on $\mathrm{MOH}$}

Density-functional theory (DFT) calculations were carried out on the following conformations as shown in Fig. 3: (a) initial, (b) deprotonated, (c) protonated, and (d) zwitterionic states. Geometry optimization was carried out using the density-functional method with the B3LYP hybrid functional ${ }^{12,13}$ together with $6-31+G(d)$ basis set, using the GAUSSIAN 03 suite of programs. ${ }^{14}$ Spectroscopic calculations were then carried out on the optimized geometries based on the time-dependent density-functional theory (TD-DFT) using the $6-31+G(d)$ basis set.

\section{RESULTS AND DISCUSSION \\ Electronic Characterization of MO \\ Crystal Structure of Solvated MO}

Table I details the crystallographic parameters of solvated MO. The crystal system is orthorhombic and the space group is $P 2_{1} 2_{1} 2_{1}{ }^{6}{ }^{6}$ Figure 4 shows the ORTEP plot of the monomeric unit of solvated MO. The asymmetric unit includes three MO and three dioxane molecules. The dioxane molecules are here coordinating solvents which work as the ligands to the $\mathrm{Na}$ atom. These constitute three kinds of $\mathrm{Na}(\mathrm{I})$-complexes, two of which are seven-coordinate and the other is five-coordinate. As shown in Figure 5, there are two kinds of seven-coordinate complexes, one of which is chelated by six $\mathrm{O}$ atoms from the sulfonic group of four different MOs and also by one $\mathrm{O}$ atom from dioxane. The other is coordinated to seven $\mathrm{O}$ atoms from the sulfonic group of five different MOs. The five-coordinate complex includes three $\mathrm{O}$ atoms from the sulfonic group of three different MOs as well as two $\mathrm{O}$ atoms from the two different dioxanes. It is also important to note that the $\mathrm{N} / \mathrm{N}$ bond lengths are typical of the azo group $(-\mathrm{N}=\mathrm{N}-)$ but these are significantly different: $1.259(4) \AA$ for N1/N2, 1.196(5) $\AA$ for N4/ $\mathrm{N} 5$, and 1.253(4) $\AA$ for N7/N8. The monomeric unit is extended alternately to form a one dimensional polymer along the $a$ axis as shown in Figure 6. The present polymer formation ensures the thermal stability of solvated MO.

\section{Absorption Spectrum in Solution and Polarized Reflection} Spectra in the Solid State

The solid-state spectra presented below will be discussed in terms of excitonic interactions that appear between excited molecules in the crystal. ${ }^{15}$ This interaction is especially pronounced when the molar extinction coefficient is sufficiently high as in the case of dye molecules and the molecules are arranged periodically. The interaction energy $\left(\Delta E_{\text {exciton }}\right)$ is given by the dipole-dipole equation: $\Delta E_{\text {exciton }}=|\mu|^{2}\left(1-3 \cos ^{2} \theta\right) / r^{3}$, where $\mu$ denotes the transition dipole, $r$ the distance between two central points of two dipoles and $\theta$ the angle between the one dipole and the vector connecting the two point dipoles. The geometrical

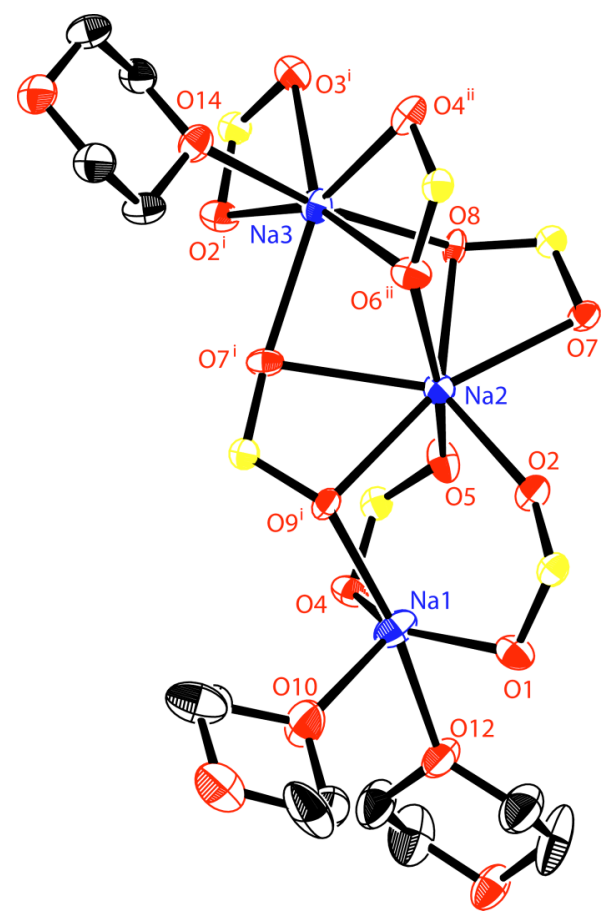

Figure 5. Three kinds of $\mathrm{Na-complexes:} \mathrm{five-coordinate} \mathrm{Nal}$ and sevencoordinate $\mathrm{Na} 2$ and $\mathrm{Na} 3$. 


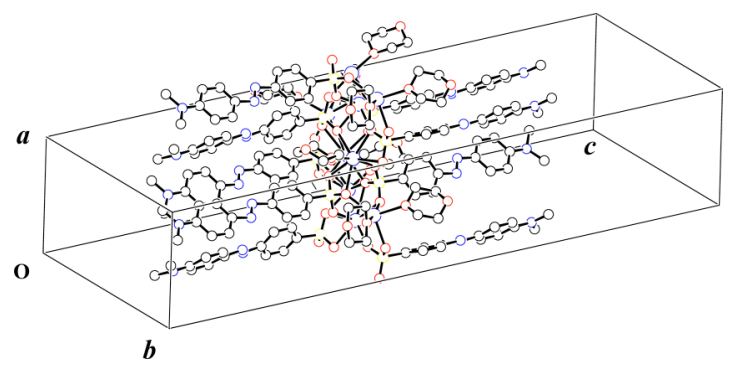

Figure 6. Crystal packing of dioxane-coordinated $M O$

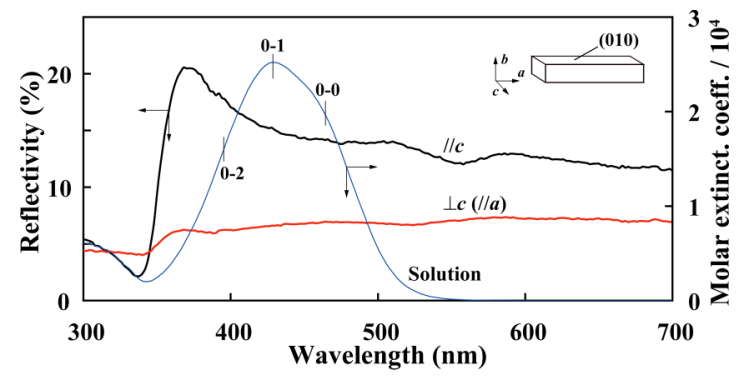

Figure 7. Absorption spectrum in solution, and the polarized reflection spectra measured on the $(\mathrm{O} 10)$ plane of $\mathrm{MO}$ single crystals. The direction of the transition dipole points along the long molecular axis as shown in Fig. 1

term $\left[\left(1-3 \cos ^{2} \theta\right) / r^{3}\right]$, which is related to the crystal structure, determines the direction of the spectral shift; whereas the extent of the spectral shift is governed by the term $|\mu|^{2}$ called the interneighbor coupling that depends directly on the absorption coefficient of the molecule. The J-aggregatelike bathochromic or $\mathrm{H}$-aggregate-like hypsochromic shift depends on the critical angle of $\theta=54.7^{\circ}$, below which the former will result and above which the latter will be the case. This interaction, however, falls off as the inverse cube of distance, so the most of the interaction comes from nearest neighbors. This means that it is qualitatively sufficient to discuss only the two neighboring molecules.

Figure 7 shows the absorption spectrum of $\mathrm{MO}$ in solution and the polarized reflection spectra measured on the (010) plane of dioxane-coordinated MO single crystals (see Figs. 4 and 6). The absorption spectrum in solution exhibits a broad band in the wavelength region between 350 and 500 $\mathrm{nm}$. However, the vibronic bands such as $0-0,0-1$, and $0-2$ transition can still be assigned as denoted by arrows, where the $0-1$ transition gives the most intensive band. In the solid state, a prominent band appears around $373 \mathrm{~nm}$ for polarization parallel to the $c$ axis which is nearly along the direction of the transition dipole [Fig. 1(a)]. On the other hand, polarization perpendicular to the $c$ axis (i.e., parallel to the $a$ axis) quenches mostly the reflection band. The band around $373 \mathrm{~nm}$ is significantly displaced toward shorter wavelengths as compared with the absorption band in solution. This is due presumably to excitonic interactions between closely overlapped two molecules shown in Fig. $6 .{ }^{15}$

\section{Electronic Characterization of $\mathrm{MOH}$}

Crystal Structure of $\mathrm{MOH}$

Table II details the crystallographic parameters of the purple phase (i.e., the new phase) together with those of the vivid-
Table II. Crystallographic parameters for the purple and vivid-red phases.

\begin{tabular}{lcc}
\hline & Purple phase & Vivid-red phase ${ }^{a}$ \\
\hline Molecular formula & $\mathrm{C}_{14} \mathrm{H}_{15} \mathrm{~N}_{3} \mathrm{O}_{3} \mathrm{~S}_{1} \cdot \mathrm{H}_{20} \mathrm{O}_{10}$ & $\mathrm{C}_{14} \mathrm{H}_{15} \mathrm{~N}_{3} \mathrm{O}_{3} \mathrm{~S}_{1}$ \\
Crystal system & Tetragonal & Monoclinic \\
Space group & $14 / \mathrm{m}$ & $P 2_{1}$ \\
$Z$ & 8 & 2 \\
$a(\AA)$ & $25.598(3)$ & $7.290(4)$ \\
$b(\AA)$ & $25.598(3)$ & $7.551(4)$ \\
$c(\AA)$ & $6.8491(8)$ & $12.727(6)$ \\
$\beta\left({ }^{\circ}\right)$ & & $104.447(9)$ \\
$V\left(\AA^{3}\right)$ & $4488.0(8)$ & $678.4(6)$ \\
$D_{x}\left(\mathrm{~g} / \mathrm{cm}^{3}\right)$ & 1.434 & 1.495 \\
$R_{1}$ & 0.16 & 0.0471 \\
${ }^{0}$ Reference 9. & &
\end{tabular}

red phase (i.e., the zwitterionic phase ${ }^{9}$ ). The structure analysis of the purple phase is not yet entirely refined at the moment. Especially, water molecules seem to fill voids in the crystal lattice as described below and can appear in several different orientations in the same voids of different unit cells of the crystal, giving rise to diffuse scattering of the bulk solvent. Therefore, it should be borne in mind that the $R$ value of the analysis is still as high as approximately 0.16 . Nevertheless, it is hopefully sufficient to discuss the molecular arrangement for the interpretation of the polarized reflection spectra measured on single crystals.

The ORTEP plot of the purple phase is shown in Figure $8(a)$. The present structure reveals that the $\mathrm{N}=\mathrm{N}$ bond length is 1.24(3) $\AA$, which is typical of the azo group $(-\mathrm{N}=\mathrm{N}-)$. Furthermore, it is to be noted that one of the $\mathrm{S} / \mathrm{O}$ bonds is apparently longer $[1.60(2) \AA]$ than the other two bonds [1.37(3) and 1.37(3) $\AA$ ], suggesting the typical structure of the sulfonic group $\left(-\mathrm{SO}_{3} \mathrm{H}\right)$.

Fig. 8(b) shows the molecular arrangement of the molecules projected onto the (001) plane. Two molecules are nearly directly overlapped. There is a column of voids in the center of the unit cell down the $c$ axis. We assume that ten water molecules fill the void in the unit cell, as calculated from our density measurement of $\mathrm{MOH}$ single crystals based upon floating method. Normally, the measured density $\left(D_{m}\right)$ is smaller than the x-ray one $\left(D_{x}\right)$ by about $5 \%$. The density measured was $1.37 \mathrm{~g} / \mathrm{cm}^{3}$. Correction gives a value of $1.44 \mathrm{~g} / \mathrm{cm}^{3}$ which is in good agreement with the $D_{x}$ shown in Table II. The proposed formula is $\mathrm{C}_{14} \mathrm{H}_{15} \mathrm{~N}_{3} \mathrm{O}_{3} \mathrm{~S} /\left(\mathrm{H}_{2} \mathrm{O}\right)_{10}$.

Spectroscopic Calculations for Neutral, Deprotonated, Protonated, and Zwitterionic Configurations of $\mathrm{MOH}$ Spectroscopic calculations were carried out for various ionic states of $\mathrm{MOH}$ in order to compare their results with those of experiments. Table III shows the absorption bands and the $\mathrm{N}=\mathrm{N}$ bond lengths for the neutral, deprotonated, protonated, and zwitterionic structures (Fig. 3). The transition dipole of the molecule in these structures is also found to 


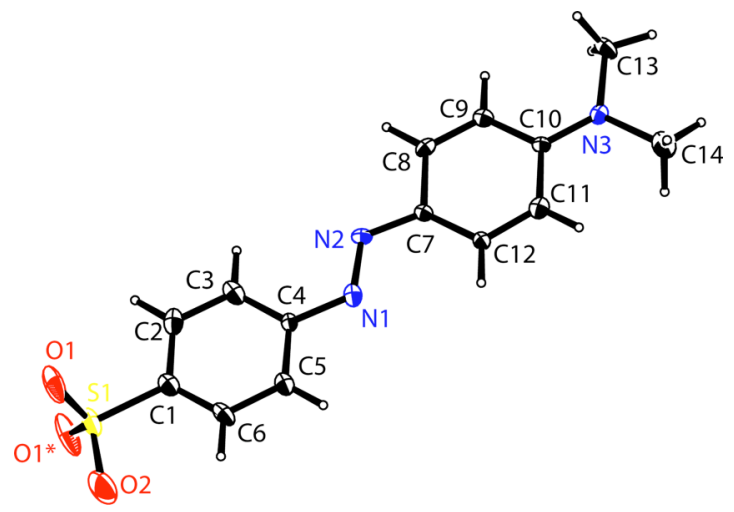

(a)

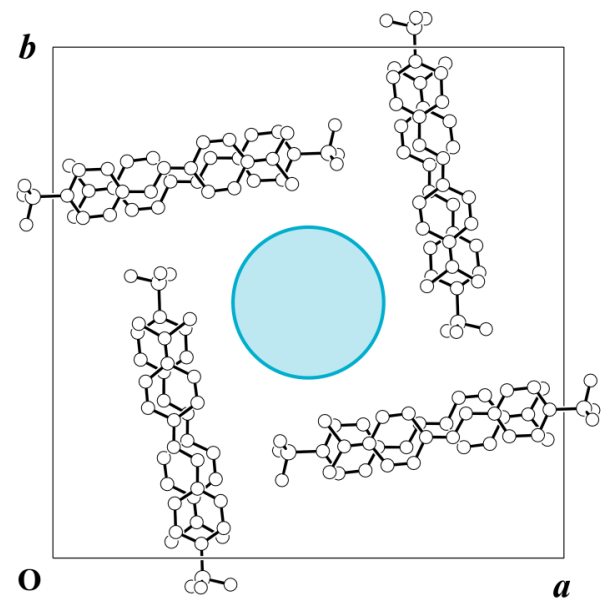

(b)

Figure 8. (a) Molecular conformation of the purple phase and (b) molecular arrangement. The two molecules are closely overlapped with an interplanar distance of about $3.4 \mathrm{~A}$ and with a slip angle of about $71^{\circ}$.

Table III. Calculated N=N bond lengths and optical absorption.

\begin{tabular}{lccc}
$\mathrm{MOH}$ & $\begin{array}{c}\mathrm{N}=\mathrm{N} \\
(\AA)\end{array}$ & $\begin{array}{c}\lambda \\
(\mathrm{nm})\end{array}$ & Oscillator strength \\
\hline (a) Initial state & 1.266 & 419.1 & 1.044 \\
(b) Deprotonated state & 1.264 & 421.6 & 0.859 \\
(c) Protonated state & 1.289 & 453.7 & 1.165 \\
(d) Zwitterionic state & 1.282 & 658.5 & 0.643 \\
\hline
\end{tabular}

point along the long-molecular axis. The $\mathrm{N}=\mathrm{N}$ bond around $1.26-1.29 \AA$ is nearer to the double bond of $1.24 \AA$ rather than to the $\mathrm{N}-\mathrm{N}$ single bond of $1.44 \AA$, suggesting that the molecule is of the azo type. Deprotonation brings about no noticeable spectral shift $(419.1 \rightarrow 421.6 \mathrm{~nm})$; whereas a significant bathochromic displacement is observed on protonation $(419.1 \rightarrow 453.7 \mathrm{~nm})$. Furthermore, a drastic bathochromic shift occurs with the zwitterionic structure $(419.1 \rightarrow 658.5 \mathrm{~nm})$.

Experiments for Deprotonation and Protonation of $\mathrm{MOH}$ in Solution

The absorption spectra were measured over the course of the deprotonation or protonation process in combination with

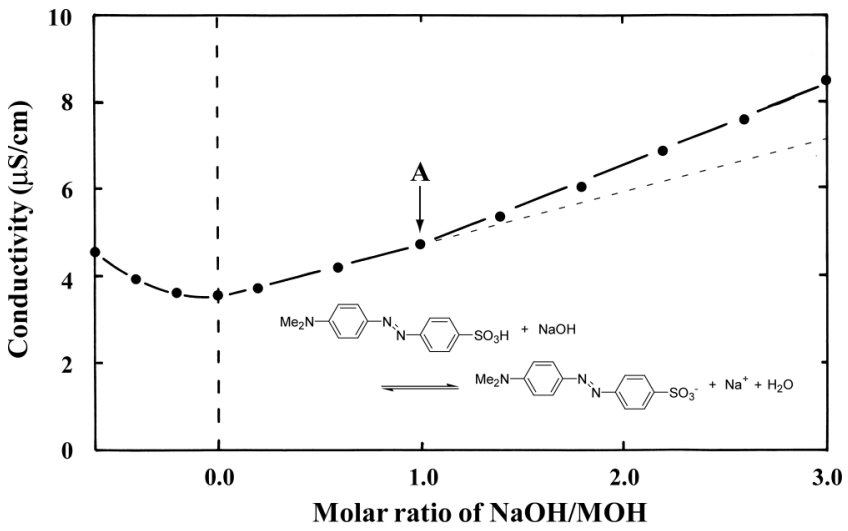

(a)

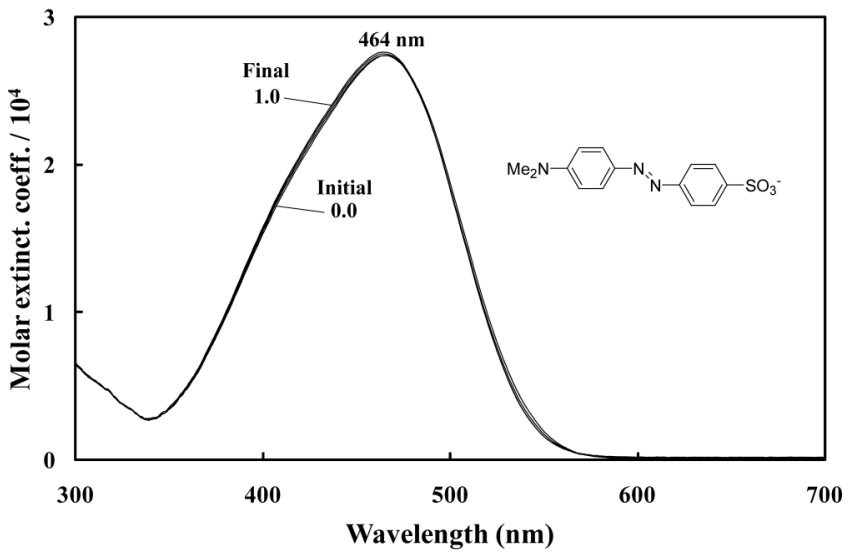

(b)

Figure 9. (a) Conductmetric titration of $\mathrm{MOH}$ aqueous solution $\left(2 \times 10^{-5} \mathrm{M} ; 100 \mathrm{ml}\right)$ with $\mathrm{NaOH}$ and (b) solution spectra of $\mathrm{MOH}$ upon deprotonation.

conductometric titration. Figure 9(a) shows the conductometric titration of $\mathrm{MOH}$ with $\mathrm{NaOH}$ for deprotonation of $\mathrm{MOH}$. The conductivity of the solution increases linearly from the commencement of the titration. This represents the neutralization of the acid $(\mathrm{MOH})$. From point A, the conductivity increases with a higher gradient. Point A corresponds exactly to the equivalence point (i.e., complete deprotonation). The absorption spectra from the initial point to point A are shown in Fig. 9(b), where the absorption maximum is present around $464 \mathrm{~nm}$. This hypsochromic shift is very small and this trend is well reproduced in the results of DFT calculations (Table III).

Figure 10(a) shows the conductometric titration for protonation of $\mathrm{MOH}$. In contrast to the quantitative titration in deprotonation experiment, roughly 30 times more $\mathrm{HCl}$ is necessary for the protonation of $\mathrm{MOH}$ due to solvation with water molecules. Fig. 10(b) shows the spectral changes during the protonation, characterized by two isosbestic points at 350 and $470 \mathrm{~nm}$. A relatively large bathochromic shift of about $30 \mathrm{~nm}$ is observed upon protonation. This tendency agrees fairly well with the calculated absorption bands (Table III).

As for the zwitterionic structure of $\mathrm{MOH}$, no experimental result is available in solution, since the zwitterionic structure exists only in the solid state. However, the fact that 


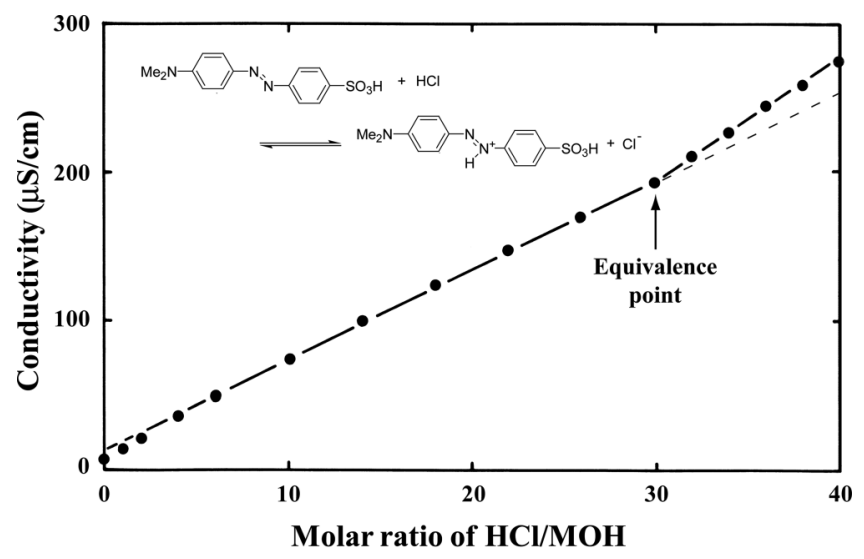

(a)

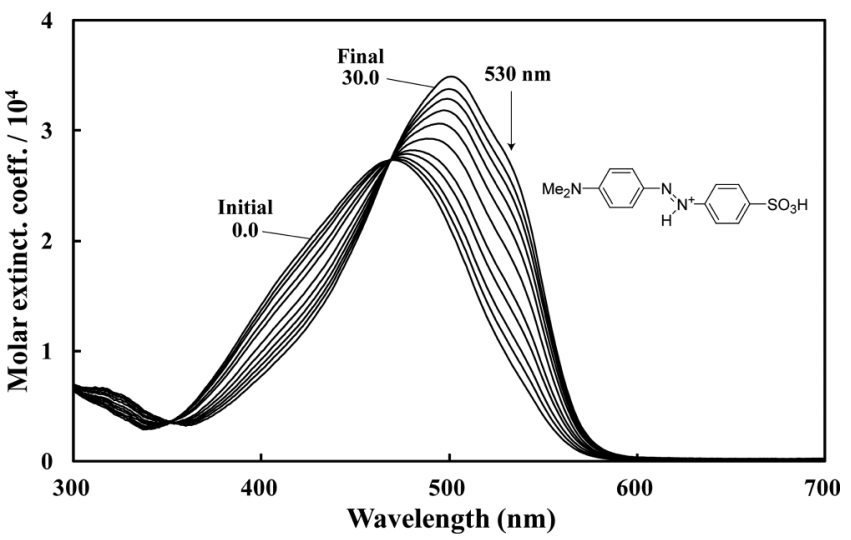

(b)

Figure 10. (a) Conductmetric titration of $\mathrm{MOH}$ aqueous solution $\left(2 \times 10^{-5} \mathrm{M} ; 100 \mathrm{ml}\right)$ with $\mathrm{HCl}$ and (b) solution spectra of $\mathrm{MOH}$ upon protonation.

the DFT calculations for deprotoantion and protonation generally agree fairly well with experiment suggests that calculation is also reliable for the zwitterionic structure.

\section{Polarized Reflection Spectra Measured on Single Crystals of $\mathrm{MOH}$}

Figure 11 shows the polarized reflection spectra for the red phase (i.e., the zwitterionic phase $\left.{ }^{9}\right)$ measured on the (001) plane of single crystals. A prominent reflection band appears

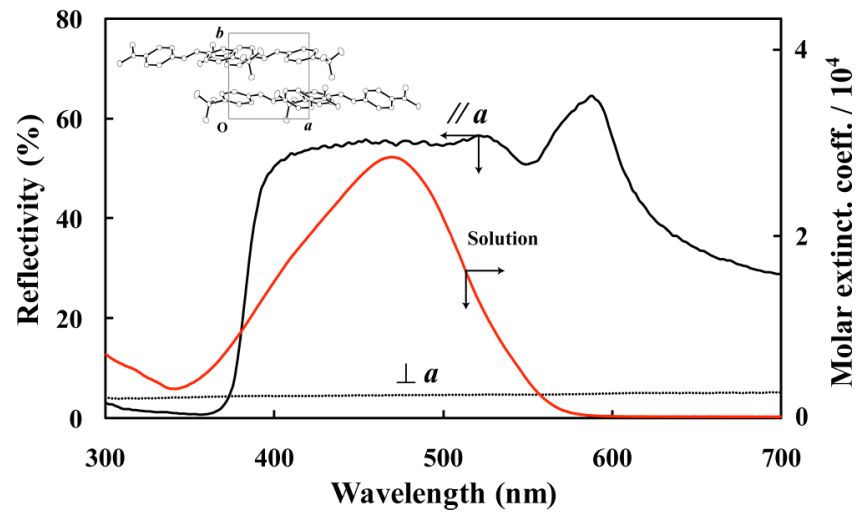

Figure 11. Polarized reflection spectra measured on the $(001)$ plane of $\mathrm{MOH}$ single crystals of the red phase together with the solution spectrum.

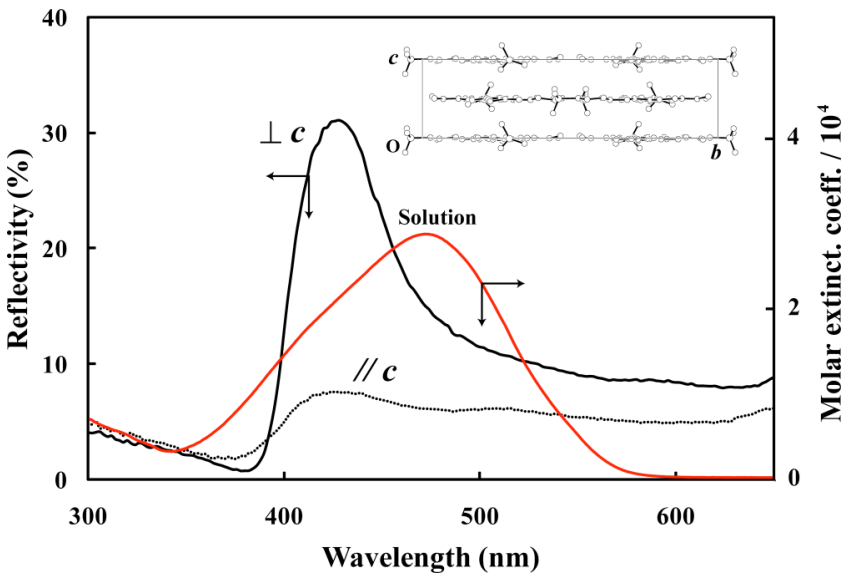

Figure 12. Polarized reflection spectra measured on the (100) plane of $\mathrm{MOH}$ single crystals of the purple phase together with the solution spectrum.

around $590 \mathrm{~nm}$ for polarization parallel to the $a$ axis, that is, the direction of the long-molecular axis. On the other hand, the reflection band is completely quenched for polarization perpendicular to the $a$ axis. These results clearly indicate that the direction of the transition dipole points along the longmolecular axis in accord with direction deduced from DFT calculations (Fig. 1). It is also important to note that the reflection spectra are quite different from the solution spectrum, especially at the longest-wavelength band around 590 $\mathrm{nm}$. This band is presumably attributed to the zwitterionic structure. As shown in Table III, the DFT calculation estimates an absorption band around $658.5 \mathrm{~nm}$ which is longer wavelength by more than $200 \mathrm{~nm}$ than the maxima associated with the initial, deprotonated, and protonated states. This result would support the above spectral assignment.

Figure 12 shows the polarized reflection spectra for the purple phase measured on the (100) plane for polarization parallel or perpendicular to the $c$ axis. A prominent reflection band appears around $420 \mathrm{~nm}$ which yields a purple color. The present band is apparently displaced shorter wavelength as compared with the absorption band in solution. The present hypsochromic shift upon crystallization can presumably be attributed to excitonic interactions between two closely overlapped molecules along the stacking $c$ axis. $^{15}$

\section{CONCLUSIONS}

The crystal and electronic structure of the tri-nuclear azo-Na complex of MO as well as of the two phases of $\mathrm{MOH}$, with special attention to the zwitterionic structure of $\mathrm{MOH}$. The conclusions can be summarized as follows:

(1) The trinuclear azo-Na complex of MO crystallizes in a space group of $P 2{ }_{1} 2_{1} 2_{1}$ and includes three $\mathrm{MO}$ and three dioxane molecules. The monomeric unit is extended to form a one dimensional polymer along the $a$ axis. The $\mathrm{N} / \mathrm{N}$ bond $(1.20-1.26 \AA)$ is typical of the azo structure $(-\mathrm{N}=\mathrm{N}-)$. The reflection band appears around $373 \mathrm{~nm}$ in the solid state and is significantly displaced toward shorter wavelengths as compared with the absorption band in 
solution around $431 \mathrm{~nm}$. This is attributed to excitonic interactions between two closely overlapped molecules in the crystal.

(2) The red and purple crystals of $\mathrm{MOH}$ were simultaneously grown in the same pot. The vivid-red crystal (space group: $\left.P 2_{1}\right)^{9}$ is characterized by the zwitterionic structure formed by $\mathrm{NH} \cdots \mathrm{O}$ intermolecular hydrogen bonds; whereas the purple phase (space group: $I 4 / \mathrm{m}$ ) is found to include ten water molecules in the asymmetric unit. The zwitterionic structure gives a N/N bond length of about $1.308 \AA$, suggesting slightly a hydrazonelike structure. In addition, the zwitterionic structure is found to drastically displace the absorption band toward longer wavelengths. This makes the color vivid red in the solid state. On the other hand, the $\mathrm{N} / \mathrm{N}$ bond is $1.24(3) \AA$ and is typical of the azo structure $(-\mathrm{N}=\mathrm{N}-)$. The purple color can basically be interpreted as arising from the hypsochromic shift upon crystallization caused by excitonic interactions between closely overlapped two molecules.

\section{REFERENCES}

${ }^{1}$ M. Herbst and K. Hunger, Industrial Organic Pigments, 3rd ed. (VCH, Weinheim, 2004)

${ }^{2}$ A. Whitaker, "The crystal structure of a second polymorph $(\beta)$ of C. I. pigment red 1, 1-[(4-nitrophenyl)azo]-2-naphthol", Z. Kristallogr. 152, $227-238$ (1980).
${ }^{3}$ A. Whitaker, "Crystal structure snalysis of azo pigments involving $\beta$-naphthol: A review”, J. Soc. Dyers Colour. 94, 431-435 (1978).

${ }^{4} \mathrm{~A}$. Whitaker, "Crystal structure of azo pigments derived from acetoacetanilide", J. Soc. Dyers Colour. 104, 294-300 (1988).

${ }^{5} \mathrm{~A}$. Whitaker, "Crystal structure of azo colorants derived from pyrazolone: A review”, J. Soc. Dyers Colour. 111, 66-72 (1995).

${ }^{6} \mathrm{~K}$. Sato, H. Shibata, and J. Mizuguchi, "Poly[tris $\left\{\mu_{2}-4-[4-\right.$ (dimethylamino)phenyldiazenyl]benzene-sulfonate\}

tridioxanetrisodium(I)]", Acta Crystallogr., Sect. B: Struct. Sci. E64, 586-587 (2008).

${ }^{7}$ A. W. Hanson, "The crystal ctructure of methyl orange monohydrate monoethanolate", Acta Crystallogr., Sect. B: Struct. Crystallogr. Cryst. Chem. 29, 454-460 (1973).

${ }^{8}$ A. R. Kennedy, J. B. A. Kirkhouse, K. M. McCarney, O. Puissegur, W. E. Smith, E. Staunton, S. J. Teat, J. C. Cherryman, and R. James, "Supramolecular motifs in $s$-block metal-bound sulfonated monoazo dyes, Part 1: Structural class controlled by cation type and modulated by sulfonate aryl ring position", Chem.-Eur. J. 10, 4606-4615 (2004).

${ }^{9}$ N. J. Burke, A. D. Burrows, M. F. Mahon, S. J. Teat, and J. Simon, "Incorporation of sulfonate dyes into hydrogen-bonded networks", Cryst. Eng. Comm. 6, 429-436 (2004).

${ }^{10}$ M. C. Burla, R. Caliandro, M. Camalli, B. Carrozzini, G. L. Cascarano, L. DeCaro, C. Giacovazzo, G. Polidor, and R. Spagna, "SIR2004: An improved tool for crystal structure determination and refinement", J. Appl. Crystallogr. 38, 381-388 (2005).

${ }^{11}$ G. M. Sheldrick, "A short history of SHELX", Acta Crystallogr., Sect. A: Found. Crystallogr. 64, 112-122 (2008).

${ }^{12}$ A. D. Becke, "Density-functional thermochemistry. III. The role of exact exchange", J. Chem. Phys. 98, 5648-5652 (1993).

${ }^{13}$ C. Lee, W. Yang, and R. G. Parr, "Development of the Colle-Salvetti correlation-energy formula into a functional of the electron density", Phys. Rev. B 37, 785-789 (1988).

${ }^{14}$ GAUSSIAN 03, revision D.01., Gaussian, Inc. (2004).

${ }^{15}$ D. P. Craig and S. H. Walmsley, Excitons in Molecular Crystals: Theory and Aapplications (Benjamin, New York, 1968). 
(C)2009 Society for Imaging Science and Technology (IS\&T)

All rights reserved. This paper, or parts thereof, may not be reproduced in any form without the written permission of IS\&T, the sole copyright owner of The Journal of Imaging Science and Technology.

For information on reprints or reproduction contact

Donna Smith

Production Editor

The Journal of Imaging Science and Technology

Society for Imaging Science and Technology

7003 Kilworth Lane

Springfield, Virginia 22151 USA

703/642-9090 extension 107

703/642-9094 (fax)

dsmith@imaging.org

www.imaging.org 